\title{
Evaluating the opioid epidemic: a urologist's promise to curb the crisis
}

\author{
Ram Anil Pathak, Ashok Kumar Hemal \\ Wake Forest University Baptist Medical Center, Boulevard, Winston-Salem, NC, USA \\ Correspondence to: Ram Anil Pathak. Wake Forest University Baptist Medical Center, Boulevard, Winston-Salem, NC, USA. \\ Email: rpathak@wakehealth.edu. \\ Comment on: Welk B, McClure JA, Clarke C, et al. An opioid prescription for men undergoing minor urologic surgery is associated with an increased \\ risk of new persistent opioid use. Eur Urol 2020;77:68-75.
}

Submitted Apr 28, 2020. Accepted for publication Sep 27, 2020.

doi: $10.21037 /$ tau-2020-899

View this article at: http://dx.doi.org/10.21037/tau-2020-899

Acute overdose due to opioid ingestion is a leading cause of emergency room visits in North America. Contrary to popular belief, most opioid abuse derives from prescription medication rather than illicit drug use (1). The exact etiology of opioid abuse stems from not taking the medication as prescribed or by using opioids that were allotted to another patient. The misuse of opioids has spawned the CDC to label it the "fastest growing drug problem in the United States". In fact, overdose secondary to opiate medication has dramatically increased since 1999, with 6-fold increase in the number of deaths occurring in 2017 (2). Therefore, adaptation by the physician, as the gatekeeper of prescriptions, represents an integral component to circumvent this growing epidemic.

Welk et al. indirectly defined the addictive nature of opioid medication in their manuscript entitled "An opioid prescription for men undergoing minor urologic surgery is associated with an increased risk of new persistent opioid use" (3). Utilizing a variety of population-level databases centralized to Ontario, Canada, the authors examined the implicit nature of opioid dependence after urologic surgery. Using minor procedures, such as transurethral prostatectomy, internal urethrotomy, and scrotal surgery as a reference, the authors measured the receipt of a second opioid prescription filled 9 to 15 months postoperatively. Key to the findings of the manuscript is that men who filled the initial opioid prescription were significantly more likely to fill a second opioid prescription and more likely to require an emergency visit or hospital admission for opioid intoxication (3). In fact, "for every 148 men who received a post-procedure opioid prescription, 1 had evidence of new persistent opioid use a year later". The authors further describe additional sensitivity analyses which were in concert with the findings from their primary and secondary objectives.

Central to the receipt of opioid prescriptions are physicians and, specifically, physician practice patterns. In the present study, urologists more often prescribed opioids after vasectomy than family physicians $(45 \%$ vs. $11 \%, \mathrm{P}<0.01)$. Moreover, the median morphine equivalent (MEQ) provided in Ontario, Canada was quite high at $113 \mathrm{mg}$; typically, $22.5 \mathrm{mg}$ MEQ is required after radical prostatectomy (4). However, solely blaming physicians for the growing opioid epidemic is careless. Only until recently has there been a coordinated government response with implementation of prescription drug monitoring programs (5).

As urologists, we have the ability to re-write the narrative. Urolithiasis represents the second most common cause of opioid prescription receipt in an analysis of emergency department visits from 2006-2017 (6). Similar to the design of the current study, post-ureteroscopy opioid practice patterns were examined with the finding that 1 in 16 opioid-naïve patients required persistent opioid use post ureteroscopy (7). A recent prospective evaluation of patients undergoing ureteroscopy found that patients can successfully be discharged without an opioid prescription (8). With respect to scrotal surgery, a prospective study found no difference between pain scores post scrotal surgery in men receiving 8 versus 4 narcotic pills (9). But what if we could limit it to 0 pills post scrotal surgery?

From a minimally-invasive surgical standpoint, we 


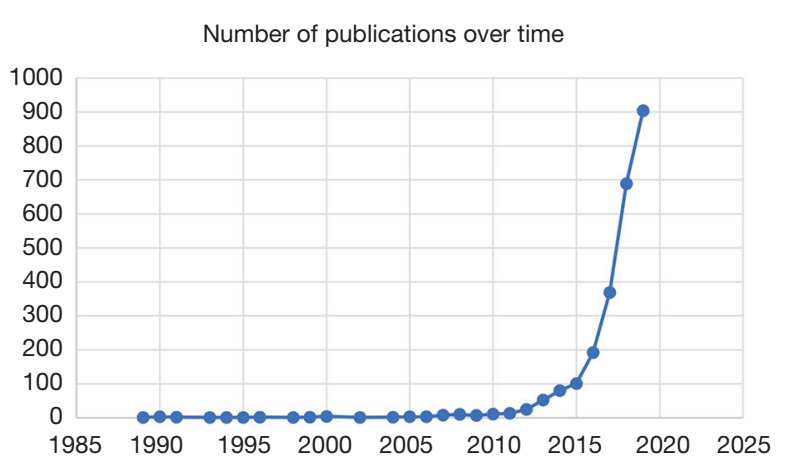

Figure 1 Using the search term 'opioid epidemic', the number of publications is charted over time.

have sought to transform our practice patterns at both the university setting and at a Veterans Affairs Medical Center. Specifically, we evaluated the feasibility of a completely opiate-free pain control regimen by measuring post-operative pain scores in patients undergoing robotic radical prostatectomy (RRP) via a pilot study. Data were prospectively collected from patients undergoing RRP. Prior to January 2019, patients received either tramadol or another opiate (either hydrocodone-acetaminophen or oxycodone-acetaminophen) post-operatively. After 2019, an opiate-free pathway including scheduled Tylenol, gabapentin, and ketorolac was instituted for all patients. Furthermore, we performed a transversus abdominis plane (TAP) block utilizing liposomal bupivacaine. Pain scores were collected on both post-operative day 0 and 1. Preliminary analysis of our data suggests feasibility of this pathway with similar pain scores in the opiate and opiate-free cohorts, demonstrating the efficacy of a totally narcotic-free regimen.

A central premise to guiding future research should encompass the following points: (I) a change in physician practice patterns, (II) patient education on the very real risks of opioid dependence, (III) alternative pain management strategies, and (IV) a new patient-physician alliance and collaborative initiative. Practice patterns can change and a slight decline in opioid initiation, approximately $0.4 \%$ over 10 years, is a reassuring finding (10). Patient education of opioid dependence is critical to limiting the epidemic. The present study found that the proportion of patients who filled an opioid prescription declined with time $37 \%$ in 2013 to $34 \%$ in $2016, \mathrm{P}<0.01$ ), which represents another encouraging finding. Alternative pain strategies should be the forefront of future research. From prostate biopsy (11) to prostatectomy (12), the whole gamut of urologic surgery should be investigated. Fortunately, a real, tangible effort to studying the 'opioid epidemic' has occurred (Figure 1). The rate of publications on PubMed has skyrocketed since 2000, a necessary focus to shed light on this crisis. Lastly, a new patient-physician alliance should be adopted, free from penalizing physicians who fail to prescribe narcotic medications with poor Press-Ganey scores or labeling every patient who requires narcotic medication as 'pain-seeking'. A thorough examination of the above would be needed to curb the growing epidemic that is the opioid crisis.

\section{Acknowledgments}

Funding: None.

\section{Footnote}

Provenance and Peer Review: This article was commissioned by the editorial office, Translational Andrology and Urology. The article did not undergo external peer review.

Conflicts of Interest: Both authors have completed the ICMJE uniform disclosure form (available at http://dx.doi. org/10.21037/tau-2020-899). Dr. AKH serves as an unpaid editorial board member of Translational Andrology and Urology from May 2019 to Apr 2021. The other author has no conflicts of interest to declare.

Ethical Statement: The authors are accountable for all aspects of the work in ensuring that questions related to the accuracy or integrity of any part of the work are appropriately investigated and resolved.

Open Access Statement: This is an Open Access article distributed in accordance with the Creative Commons Attribution-NonCommercial-NoDerivs 4.0 International License (CC BY-NC-ND 4.0), which permits the noncommercial replication and distribution of the article with the strict proviso that no changes or edits are made and the original work is properly cited (including links to both the formal publication through the relevant DOI and the license). See: https://creativecommons.org/licenses/by-nc-nd/4.0/.

\section{References}

1. Nelson LS, Juurlink DN, Perrone J. Addressing the opioid epidemic. JAMA 2015;314:1453-4. 
2. Scholl L, Seth P, Kariisa M, et al. Drug and opioidinvolved overdose deaths - United States, 2013-2017. MMWR Morb Mortal Wkly Rep 2018;67:1419-27.

3. Welk B, McClure JA, Clarke C, et al. An opioid prescription for men undergoing minor urologic surgery is associated with an increased risk of new persistent opioid use. Eur Urol 2020;77:68-75.

4. Patel HD, Srivastava A, Patel ND, et al. A prospective cohort study of postdischarge opioid practices after radical prostatectomy: the ORIOLES initiative. Eur Urol 2019;75:215-8.

5. Jones MR, Novitch MB, Sarrafpour S, et al. Government legislation in response to the opioid epidemic. Curr Pain Headache Rep 2019;23:40.

6. Rui P, Santo L, Ashman JJ. Trends in opioids prescribed at discharge from emergency departments among adults: United States, 2006-2017. Natl Health Stat Report 2020;(135):1-12.

7. Tam CA, Dauw CA, Ghani KR, et al. New persistent

Cite this article as: Pathak RA, Hemal AK. Evaluating the opioid epidemic: a urologist's promise to curb the crisis. Transl Androl Urol 2021;10(1):19-21. doi: 10.21037/tau-2020-899 opioid use after outpatient ureteroscopy for upper tract stone treatment. Urology 2019;134:103-8.

8. Gridley C, Robles J, Calvert J, et al. Enhanced recovery after surgery protocol for patients undergoing ureteroscopy: prospective evaluation of an opioid-free protocol. J Endourol 2020;34:647-53.

9. Greer AB, Ramos L, Dubin JM, et al. Decreasing postoperative opioid use while managing pain: a prospective study of men who underwent scrotal surgery. BJUI Compass 2020;1:60-3.

10. Han B, Compton WM, Jones CM, et al. Nonmedical prescription opioid use and use disorders among adults aged 18 through 64 years in the United States, 2003-2013. JAMA 2015;314:1468-78.

11. Pathak RA, Thiel DD, Parker A, et al. Timing interval from peri-prostatic block to biopsy impacts procedural pain. Can J Urol 2017;24:8795-801.

12. Theisen KM, Davies BJ. A radical proposition: opioidsparing prostatectomy. Eur Urol Focus 2020;6:215-7. 